\title{
Design of a $T$ Factor Based RBFNC for a Flight Control System
}

\author{
C. S. Mohanty, ${ }^{1}$ P. S. Khuntia, ${ }^{1}$ and D. Mitra $^{2}$ \\ ${ }^{1}$ Konark Institute of Science and Technology, Bhubaneswar 752050, India \\ ${ }^{2}$ Indian School of Mines, Dhanbad 826004, India \\ Correspondence should be addressed to C. S. Mohanty; csmkist@gmail.com
}

Received 11 February 2014; Accepted 29 March 2014; Published 24 April 2014

Academic Editor: António Dourado Pereira Correia

Copyright (C) 2014 C. S. Mohanty et al. This is an open access article distributed under the Creative Commons Attribution License, which permits unrestricted use, distribution, and reproduction in any medium, provided the original work is properly cited.

\begin{abstract}
This paper presents the design of modified radial basic function neural controller (MRBFNC) for the pitch control of an aircraft to obtain the desired pitch angel as required by the pilot while maneuvering an aircraft. In this design, the parameters of radial basis function neural controller (RBFNC) are optimized by implementing a feedback mechanism which is controlled by a tuning factor " $\alpha$ " ( $T$ factor). For a given input, the response of the RBFN controller is tuned by using $T$ factor for better performance of the aircraft pitch control system. The proposed system is demonstrated under different condition (absence and presence of sensor noise). The simulation results show that MRBFNC performs better, in terms of settling time and rise time for both conditions, than the conventional RBFNC. It is also seen that, as the value of the $T$ factor increases, the aircraft pitch control system performs better and settles quickly to its reference trajectory. A comparison between MRBFNC and conventional RBFNC is also established to discuss the superiority of the former techniques.
\end{abstract}

\section{Introduction}

The conventional design methods of a control system often require mathematical models describing the dynamic behavior of the plant to be controlled. When such mathematical models are difficult to obtain due to uncertainty or complexity, the conventional techniques based on mathematical models are not well suited. Artificial neural network (ANN) in last decade has become popular for plant identification and control $[1,2]$. An advantage of the ANN is its ability to handle the nonlinear mapping of the input-output space. It is well known that back propagation based ANN suffers from local minima and over fitting problems which is difficult to be implemented in real time due to a large number of neurons in the hidden layer in comparison to the RBFNC $[3,4]$. Since early 1990s, radial basis function network with Gaussian function has been widely used as the basic structure of neural network in nonlinear control [5-7]. Locally tuned and overlapping receptive fields have been found in cerebral cortex visual cortex and in other parts of the brain. The concept of localized information processing in the form of receptive fields suggests that such local learning offers alternative computational opportunities to learning with global basis functions [8]. Gomi and Kawato proposed a feedback error learning control strategy, where a Gaussian RBFN is used for online learning of the inverse dynamics of the system [6]. A Radial basic function neural controller (RBFNC) with learning mechanism is used to control the pitch angle of an aircraft [9]. RBFNC is used for UPFC to improve the transient stability performance of a power system [10]. The comparison between multilayer perception network (MLPN) and radial basis function network (RBFN) is done for online identification of the nonlinear dynamics of a synchronous generator [11]. Neural networks are used to build a model of the plant and to construct its "inverse" to approximate a desired model dynamics [12].

In this paper, a new modified neural controller is designed to control the pitch angle of an aircraft in various conditions and also in the presence of sensor noise. Due to the presence of $T$ factor, the weights are updated and the response of the pitch control system is accelerated and settles quickly to its reference value. 


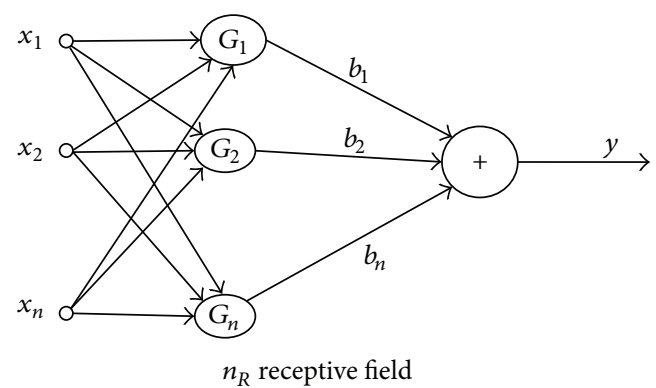

FIGURE 1: RBFNC for aircraft pitch control.

\section{Radial Basic Function Neural Network}

Radial Basis function networks are two layers feed forward networks. In radial basis function neural network (RBFNN), the hidden nodes are implementing a set of radial basis functions (e.g., Gaussian functions).

In RBFNN, the network training is divided into two stages:

(i) weights from the input to hidden layer are determined;

(ii) weights from the hidden to output layer are also determined.

The training/learning in case of RBFNN is very fast and networks are very good at interpolation. A radial basic function neural network is shown in Figure 1.

The proposed RBFNN model with single neuron output $y$ presented in Figure 1 consists of three layers [13]. Each input value is assigned to a node and passed directly to the hidden layer without weights. The hidden layer nodes are called radial basic function (RBF) units which are determined by a parameter vector called centerand a scalar called width. The Gaussian density function is used as an activation function for the hidden neurons. The RBFNN shown in Figure 1 has inputs $x_{i}, i=1,2,3, \ldots, n$, and output $y=F_{\mathrm{rbf}}(x) . x=$ $\left[x_{1}, x_{2}, x_{3}, \ldots, x_{n}\right]^{T}$ is the input and $R_{i}(x)$ is the output of the $i$ th receptive field with strength denoted by $b_{i}$. Assuming $n_{R}$ receptive fields present in the RBFNN, the output $y$ can be written as

$$
y=F_{\mathrm{rbf}}(x, \varphi)=\sum_{i=1}^{n_{R}} b_{i} R_{i}(x),
$$

where $\varphi$ holds the parameters of the receptive field units which consist of the parameters $b_{i}$ and possibly the parameters of the $R_{i}(x)$. The Gaussian-shaped functions are preferred for analytical convenience; that is,

$$
R_{i}(x)=\exp \left[\frac{\left|x-c^{i}\right|^{2}}{\left(\sigma^{i}\right)^{2}}\right]
$$

where $c_{i}=\left[c_{1}^{i}, c_{2}^{i}, \ldots, c_{n}^{i}\right]^{T}$ parameterize the locations and $\sigma$ decides the spreading of the receptive fields in the input space.

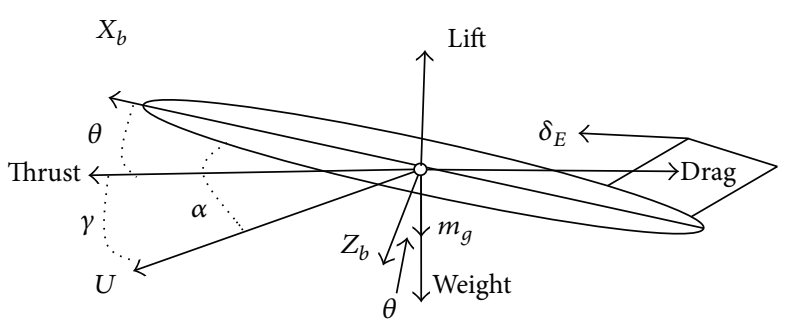

FIgURE 2: Longitudinal dynamics description of an aircraft, where $\delta_{E}=$ deflection of the elevator, $\theta=$ pitch angle, and $\alpha=$ angle of attack.

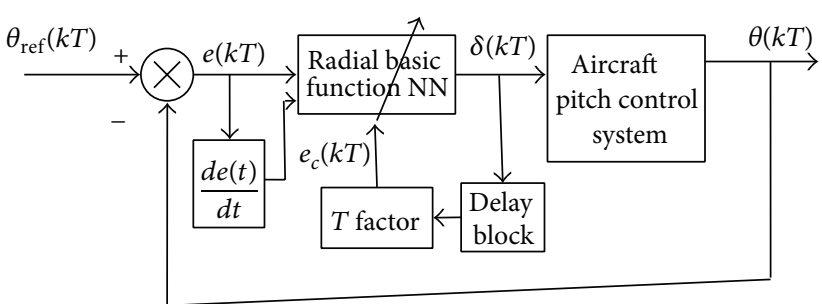

FIGURE 3: Proposed RBFNC controller for pitch control.

The weighted average output of the RBFNN can be written as

$$
y=F_{\mathrm{rbf}}(x, \varphi)=\frac{\sum_{i=1}^{n_{R}} b_{i} R_{i}(x)}{\sum_{i=1}^{n_{R}} R_{i}(x)} .
$$

\section{Mathematical Formulation of an Aircraft Pitch Control System}

The pitch angle of the aircraft is generally described by a coordinate system that is fixed to the aircraft. The pitch angle and other forces acting in an aircraft are shown in Figure 2.

The aircraft pitch control model is described by the following equation [14]:

$$
\begin{gathered}
\dddot{\theta}(t)+\left(\frac{1}{\tau_{1}}+\frac{1}{\tau_{2}}\right) \ddot{\theta}(t)+\left(\frac{1}{\tau_{1} \tau_{2}}\right) \dot{\theta}(t) \\
=\frac{k}{\tau_{1} \tau_{2}}\left(\tau_{3} \dot{\delta}_{E}(t)+\delta_{E}(t)\right) .
\end{gathered}
$$

The above equation can be represented as

$$
\frac{\theta(s)}{\delta_{E}(s)}=\frac{k\left(s \tau_{3}+1\right)}{s\left(\left(s \tau_{1}+1\right)\left(s \tau_{2}+1\right)\right)},
$$

where the values of $\tau_{1}, \tau_{2}, \tau_{3}$, and $k$ are $0.8995+0.0968 i$, $0.8995-0.0968 i, 1.0824$, and -1.1346 , respectively, for a Delta aircraft (flight condition 3) [13].

\section{Design of $T$ Factor Based RBFNC}

The RBFNC for aircraft pitch control system shown in Figure 2 tracks the desired pitch angle $\theta(k T)$. The system has a $T$ factor that is used to tune the output of the RBFNC. 


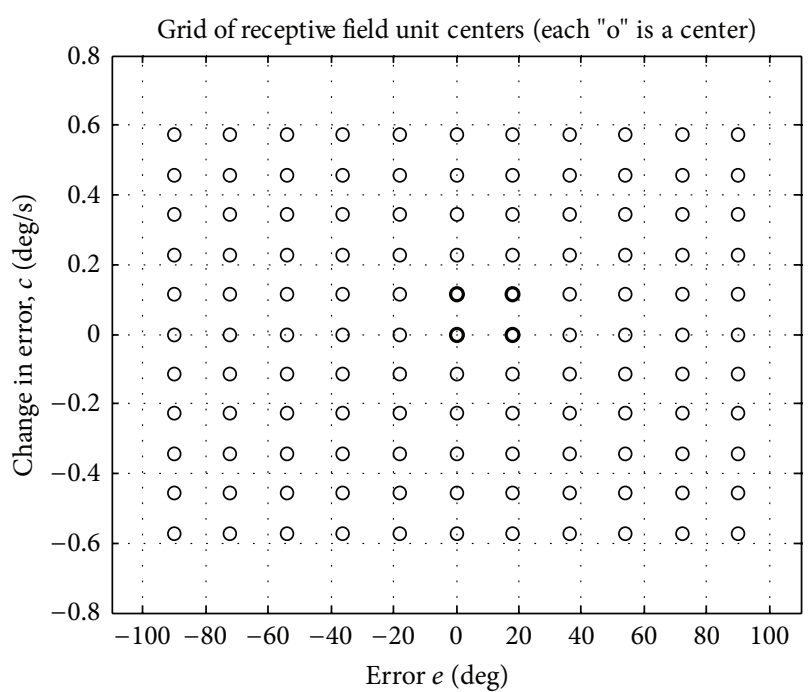

FIGURE 4: Receptive field unit centers.

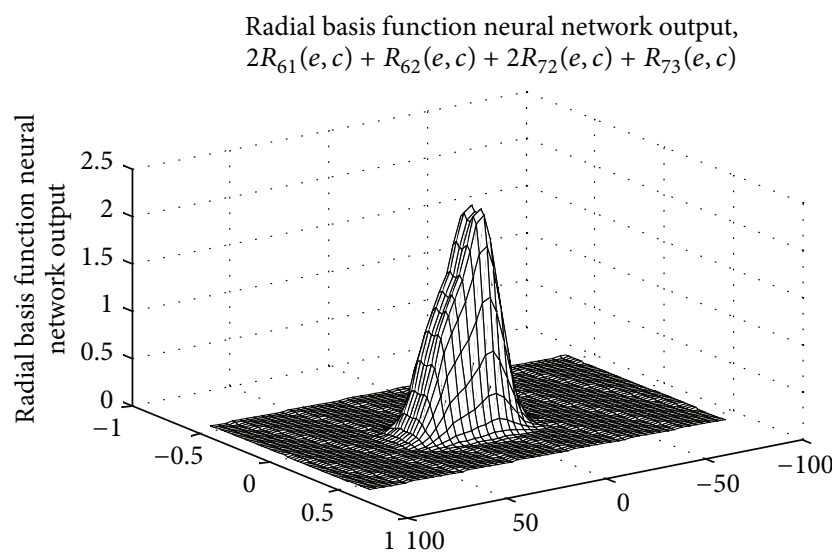

FIGURE 5: Scaling and addition of many receptive fields $2 R_{61}(e, c)+$ $3 R_{62}(e, c)+R_{72}(e, c)+2 R_{73}(e, c)$.

As shown in Figure 3, the $\alpha, e(k T), e_{c}(k T)$, and $\theta(k T)$ are used to adjust the weights of the neural controller, that is, $b_{i}$,where

$$
\begin{aligned}
e(k T) & =\theta_{\text {ref }}(k T)-\theta(k T), \\
e_{c}(k T) & =\frac{e(k T)-e(k T-T)}{T} .
\end{aligned}
$$

And $T$ is the sampling time. The output of the RBF NC $\delta(k)$ is computed by taking $e(k)$ and $e_{c}(k)$ as the argument to the radial basic function

$$
\delta(k)=F_{\mathrm{rbf}}\left(e(k), e_{c}(k), \alpha, \delta(k-1)\right),
$$

where $\alpha=T$ factor [15] and $\delta(k-1)=$ previous output of neural controller.

It is decided in the designing of pitch controller that elevator should not to exceed more than $\pi / 2$ radian in either upward or downward direction or the change of error should not be more than $0.01 \mathrm{radian} / \mathrm{sec}$. It concludes that range of

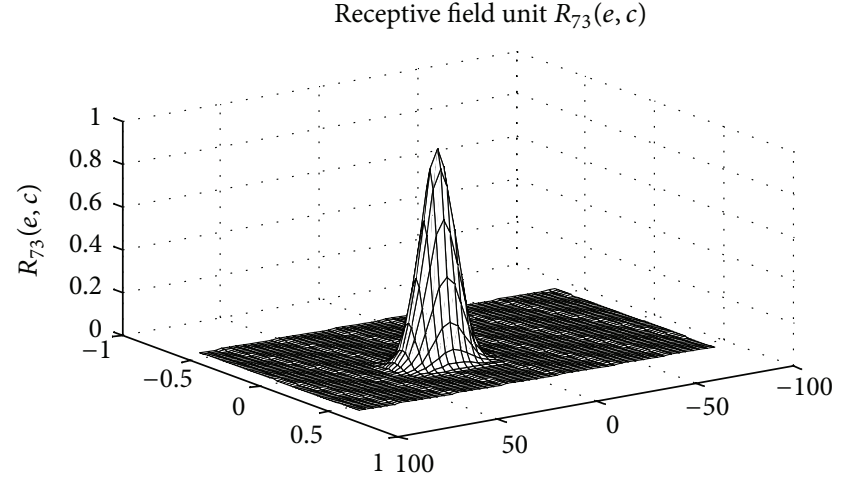

FIGURE 6: Single receptive field $R_{73}(e, c)$.

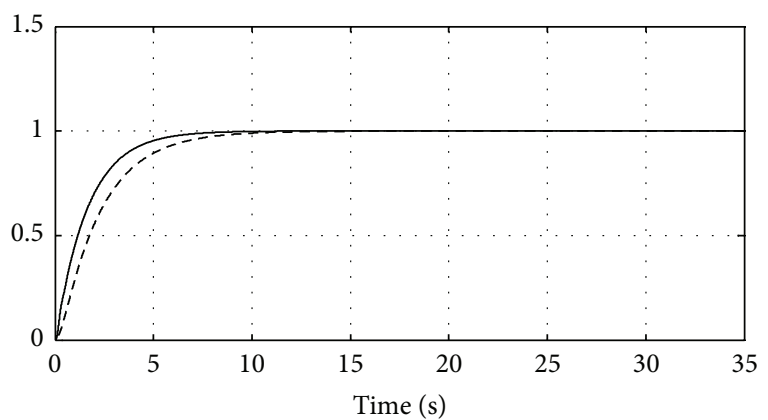

- With $T$ factor

- - - Without $T$ factor

FIGURE 7: Comparison response of pitch angle (deg) between $\alpha=0$ and $\alpha=0.9$.

error $e(k T)$ and change of error $e_{c}(k T)$ are $e(k) \in[-\pi / 2, \pi / 2]$ and $e_{c}(k) \in[-0.01,0.01]$. A uniformly grid is created by taking the error and the change of error with the corners of the grid placed at $(-\pi / 2,-0.01),(-\pi / 2,0.01),(\pi / 2,0.01)$, and $(\pi / 2,-0.01)$. Each point on the grid contains a receptive field which is a Gaussian function. The error and change of the error's spreading $(\sigma)$ are taken differently as

$$
\sigma_{c}=0.7 \frac{0.02}{\sqrt{n_{G}}}, \quad \sigma_{e}=0.7 \frac{\pi}{\sqrt{n_{R}}},
$$

where $n_{G}$ is the number of partitions on the $\operatorname{grid}\left(n_{G}=11\right.$ here) and $n_{R}$ is the number of receptive field units in RBFNC which is equal to $n_{R}=n G^{2}\left(n_{R}=121\right)$. Each center which represents a RBF is represented by circle as shown in Figure 4.

The left most bottom circle $(-\pi / 2,-0.01)$ is counted as 1 and the counting increases by 1 making the left most top circle $(-\pi / 2,0.01)$ numbered as 11 . Next counting starts from the bottom circle of the next column with number 12 and the top most circle in that column is represented as 22 . So, the right most bottom circle $(\pi / 2,0.01)$ is counted as 111 and top most circle is counted as $121(\pi / 2,-0.01)$. The input and output mapping of the radial basis function neural network is shaped by choice of scaling parameters $b_{i}$ (Figure 5). Assume the scaling and summation of the receptive field units with centers at the four dark-shaded circles shown in Figure 1 


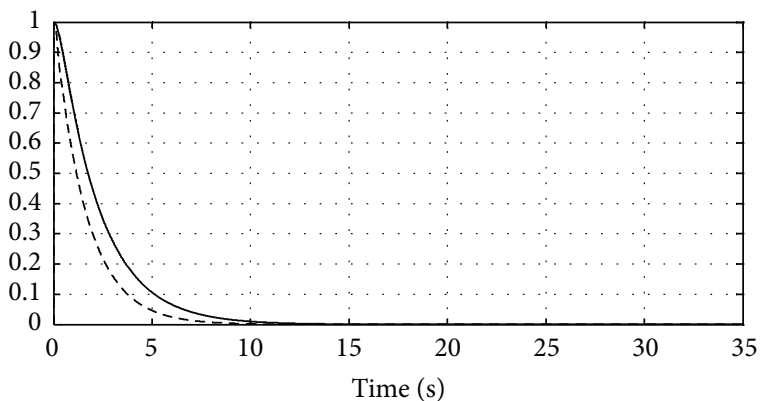

- - - With $T$ factor

- Without $T$ factor

FIGURE 8: Comparison of response of error between $\alpha=0$ and $\alpha=$ 0.9 .

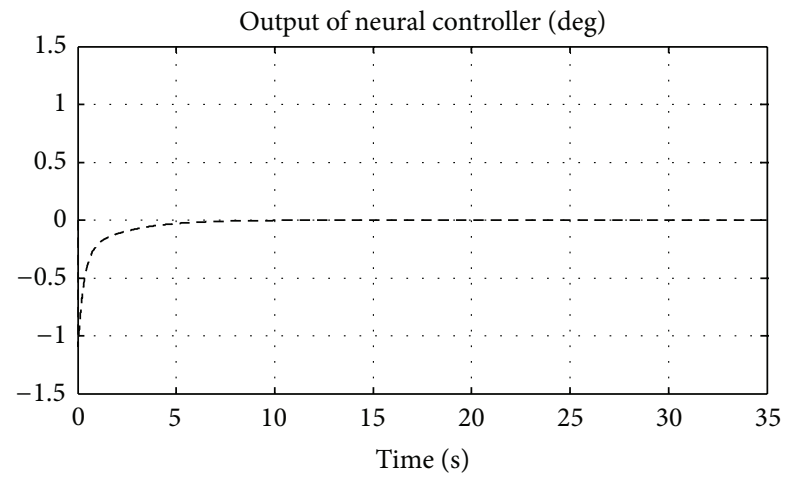

FIGURE 9: The output of RBFNC to plant for $\alpha=0$.

(the indices here are assumed to be 61, 62, 72, and 73) to be $2 R_{61}(e, c)+3 R_{62}(e, c)+R_{72}(e, c)+2 R_{73}(e, c)$. The scaling and summing are computed and shown in Figure 4.

A single receptive field $R_{73}(e, c)$ without scalingis shown in Figure 6.

For the receptive field, the parameter $\sigma$ of the Gaussian function decides the spreading of the Gaussian function. The error and change of the error's spreading $(\sigma)$ are different and are taken as

$$
\sigma_{c}=0.7 \frac{0.02}{\sqrt{n G}}, \quad \sigma_{e}=0.7 \frac{\pi}{\sqrt{n R}},
$$

where $n G$ is number of partitions on each edge of grid (here, $n G=11)$ and $n R$ is the number of receptive field units in RBF neural controller which is equal to $n R=n G^{2}$ (here, $n R=121$ ).

\section{Simulation Results}

The reference signal is a step signal and the flight travels with constant speed of $253 \mathrm{~m} / \mathrm{sec}$ of a Delta aircraft (flight condition-3). A reference pitch angle of 1 degree is given as input to the aircraft and to the reference model simultaneously. The output which is the actual pitch angle follows the reference trajectory of reference model output. The following figures illustrate the pitch angle responses of the aircraft under flight condition-3.

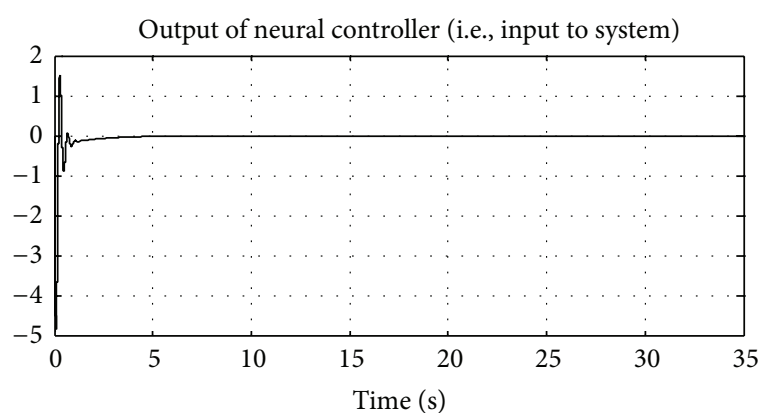

FIgURE 10: The output of RBFNC to plant for $\alpha=0.9$.

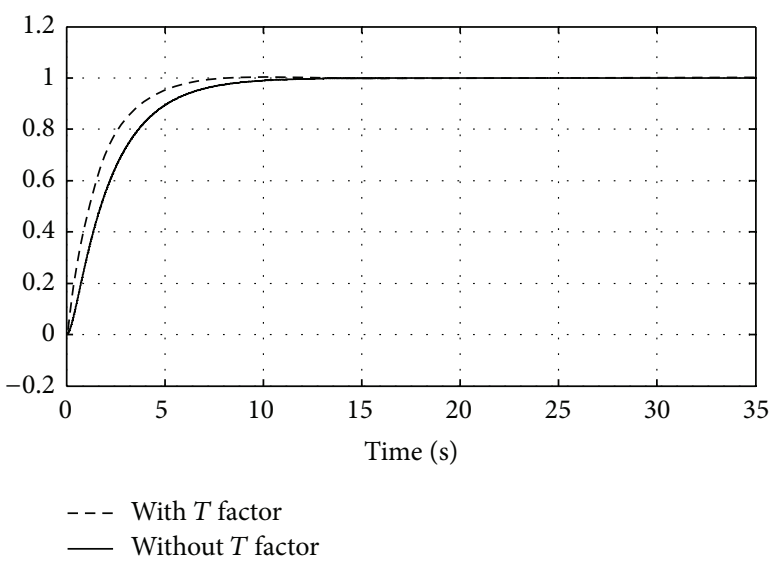

FIGURE 11: Comparison response of pitch angle (deg) for aircraft pitch control system between $\alpha=0$ and $\alpha=0.9$.

5.1. In the Absence of Sensor Noise. (1) Comparison of Closed Loop Response between $\alpha=0$ (without $T$ factor) and $\alpha=0.9$ (with $T$ factor). See Figure 7.

(2) Comparison of Pitch Angle Errors between $\alpha=0$ (without $T$ factor) and $\alpha=0.9$ (with $T$ factor). See Figure 8 .

(3) Output of Neural Controller for $\alpha=0$ (without $T$ factor). See Figure 9.

(4) Output of Neural Controller for $\alpha=0.9$ (with $T$ factor). See Figure 10.

5.2. In Presence of Sensor Noise. (1) Comparison of closed loop responses between $\alpha=0$ (without $T$ factor) and $\alpha=0.9$ (with $T$ factor). See Figure 11.

(2) Comparison of pitch angle errors between $\alpha=0$ (without $T$ factor) and $\alpha=0.9$ (with $T$ factor). See Figure 12 .

(3) Output of Neural Controller for $\alpha=0$ (without $T$ factor). See Figure 13.

(4) Output of Neural Controller for $\alpha=0.9$ (with $T$ factor). See Figure 14.

5.3. Comparison of Pitch Control System Responses for Different Values of T Factor $(\alpha=0, \alpha=0.2, \alpha=0.6$ and $\alpha=0.9)$. See Figure 15. 


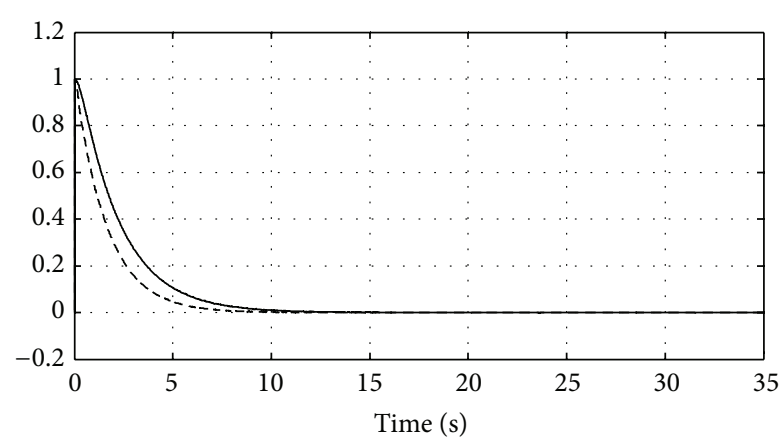

- Without $T$ factor

- - - With $T$ factor

Figure 12: Comparison of response of error between $\alpha=0$ and $\alpha=0.9$.

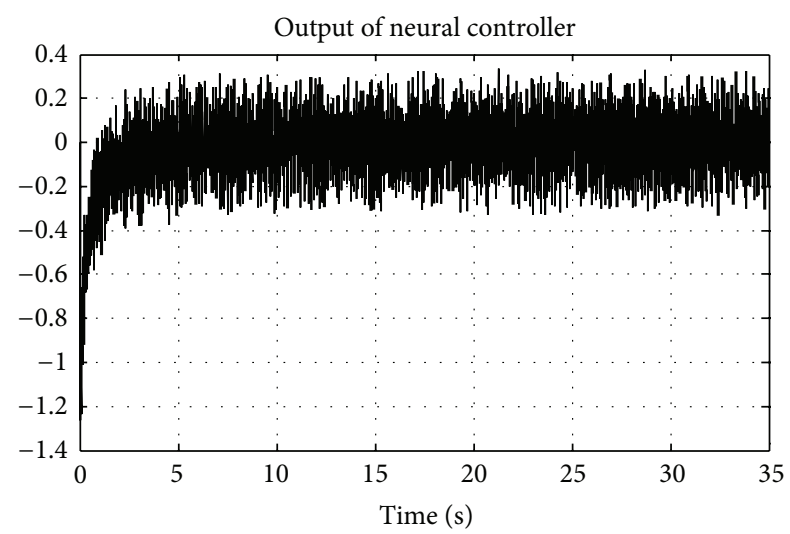

FIgURE 13: The output of RBFNC to plant for $\alpha=0$.

TABLE 1: Comparison of time domain specifications of pitch control system for different values of $\alpha$.

\begin{tabular}{lcccc}
\hline Sl. number & $\begin{array}{c}T \text { factor } \\
(\alpha)\end{array}$ & $\begin{array}{c}\text { Settling time } \\
\text { (second) }\end{array}$ & $\begin{array}{c}\text { Rise time } \\
\text { (second) }\end{array}$ & $\begin{array}{c}\text { Overshoot } \\
\text { (percentage) }\end{array}$ \\
\hline 1 & 0 & 15.84 & 6.556 & 0 \\
2 & 0.2 & 13.02 & 6.191 & 0 \\
3 & 0.6 & 10.46 & 5.401 & 0 \\
4 & 0.9 & 9.065 & 4.875 & 0 \\
\hline
\end{tabular}

\section{Conclusion}

The nonzero value of the MRBFNC output exhibits its adaptive nature whenever the actual pitch angle differs from its reference value and at the time of transition of the reference signal. When the speed of the aircraft is changed, the control signal to the pitch control system also changes to cope up with the speed change. From Table 1, it is clear that, for the value of " $\alpha=0.9$," the settling time and rise time of pitch control system are much better than the RBFNC based pitch control system. It is shown from Figures 10 and 14 that $T$ factor tunes the output of the RBFNC in both normal and sensor noise conditions. Figure 7 shows comparative analysis of closed loop response between conventional and MRBFNC.

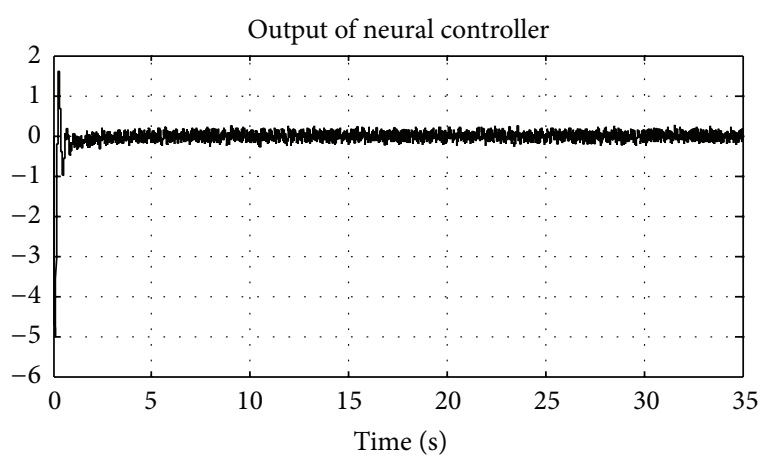

FIgURE 14: The output of RBFNC to plant for $\alpha=0.9$.

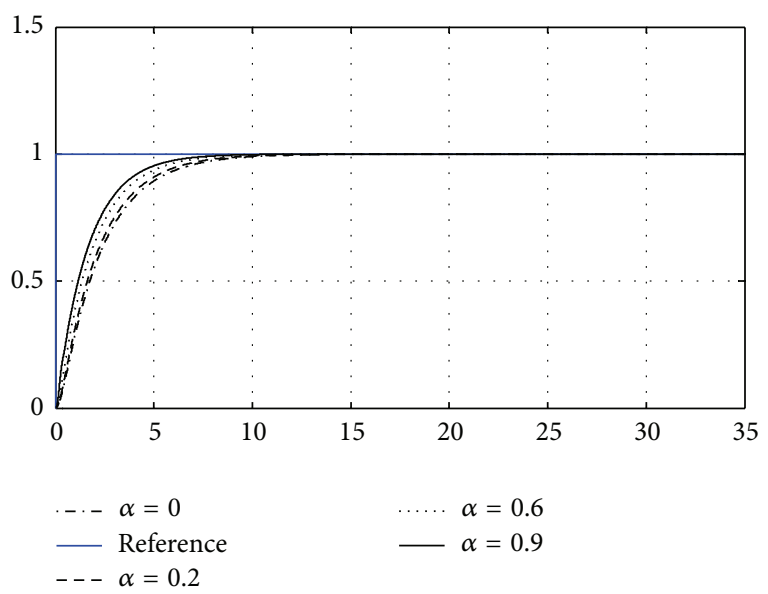

Figure 15: Comparison of closed loop responses of aircraft for different values of $T$ factors.

The $T$ factor based system has a better response and settles earlier to its references than the conventional RBFNC. Figure 15 shows the responses of the pitch control system that varies according to the value of the $T$ factor $(\alpha)$. It is concluded that, to increase the value of $\alpha$, the response $\theta(k)$ becomes settled earlier to the reference value. It is also shown in this simulation that the sensor noise does not affect the output of the RBFNC because the controller output continuously changes to nullify the effect of this noise. Considering $\alpha$ beyond 1 results in distorted value and does not follow the trajectory. This work can be further extended using a complex-valued real-time recurrent learning (CRTRL) algorithm for pitch control system. It can also be realized as fully connected recurrent neural networks [16] and can be compared with the $T$ factor based RBFNC. The current problem discussed here can also be analyzed using quaternion domain [17].

\section{Conflict of Interests}

The authors declare that there is no conflict of interests regarding the publication of this paper. 


\section{References}

[1] K. S. Narendra and K. Parthasarathy, "Identification and control of dynamical systems using neural networks," IEEE Transactions on Neural Networks, vol. 1, no. 1, pp. 4-27, 1990.

[2] S. Suresh, N. Kannan, S. N. Omkar, and V. Mani, "Nonlinear lateral command control using neural network for F-16 aircraft," in Proceedings of the American Control Conference (ACC '05), pp. 2658-2663, Portland, Ore, USA, June 2005.

[3] Y. Li, N. Sundararajan, and P. Saratchandran, "Neuro-controller design for nonlinear fighter aircraft maneuver using fully tuned RBF networks," Automatica, vol. 37, no. 8, pp. 1293-1301, 2001.

[4] M.-G. Zhang, X.-G. Wang, and M.-Q. Liu, "Adaptive PID control based on RBF neural network identification," in Proceedings of the 17th IEEE International Conference on Tools with Artificial Intelligence (ICTAI '05), pp. 681-683, November 2005.

[5] L. Yan, N. Sundararajan, and P. Saratchandran, "Stable neuroflight-controller using fully tuned radial basis function neural networks," Journal of Guidance, Control, and Dynamics, vol. 24, no. 4, pp. 665-674, 2001.

[6] H. Gomi and M. Kawato, "Neural network control for a closedloop system using feedback-error-learning," Neural Networks, vol. 6, no. 7, pp. 933-946, 1993.

[7] D. P. Mandic and J. Chambers, Recurrent Neural Networks For Prediction: Learning Algorithms, Architectures and Stability, Wiley, 2001.

[8] S. Schaal and C. G. Atkeson, "Constructive incremental learning from only local information," Neural Computation, vol. 10, no. 8 , pp. 2047-2084, 1998.

[9] P. S. Khuntia and D. Mitra, "Radial basic function neural controller for pitch control of an aircraft," Georgian Electronic Scientific Journal: Computer Science and Telecommunications, vol. 19, no. 2, 2009.

[10] P. K. Dash, S. Mishra, and G. Panda, "A radial basis function neural network controller for UPFC," IEEE Transactions on Power Systems, vol. 15, no. 4, pp. 1293-1299, 2000.

[11] J.-W. Park, R. G. Harley, and G. K. Venayagamoorthy, "Comparison of MLP and RBF neural networks using deviation signals for on-line identification of a synchronous generator," in Proceedings of the IEEE Power Engineering Society Winter Meeting, pp. 274-279, January 2002.

[12] B. Widrow and M. Bilello, "Adaptive inverse control," in Proceedings of the IEEE International Symposium on Intelligent Control, pp. 1-6, Chicago, Ill, USA, August 1993.

[13] K. M. Passino, Biomimicry For Optimization Control and Automation, Springer, London, UK, 1990.

[14] Automatic Flight Control Systems, Mclean Donald, Prentice Hall International, 1990.

[15] D. J. Swanston, J. M. Bishop, and R. J. Mitchell, "Simple adaptive momentum: new algorithm for training multilayer perceptrons," Electronics Letters, vol. 30, no. 18, pp. 1498-1500, 1994.

[16] S. L. Goh and D. P. Mandic, "A complex-valued RTRL algorithm for recurrent neural networks," Neural Computation, vol. 16, no. 12, pp. 2699-2713, 2004.

[17] C. U. Bukhari, C. Jahanchahi, C. C. Took, and D. P. Mandic, "Adaptive convex combination approach for the identification of improper quaternion processes," IEEE Transactions on Neural Networks and Learning Systems, vol. 25, no. 1, pp. 172-182, 2014. 

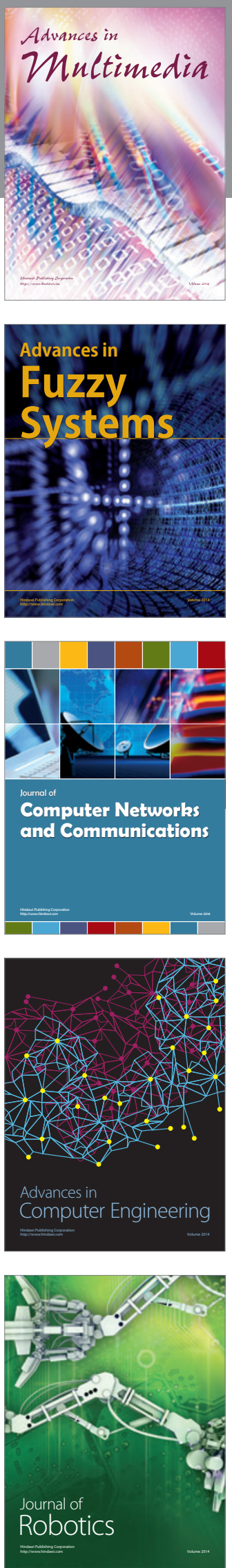

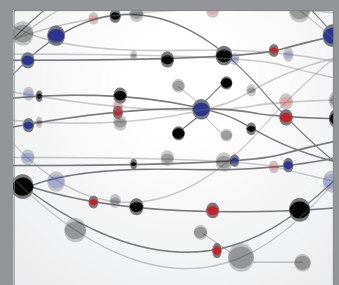

The Scientific World Journal
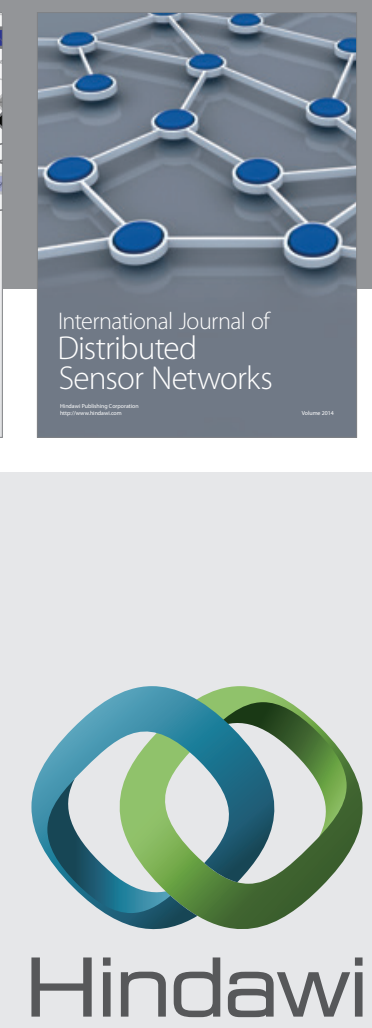

Submit your manuscripts at

http://www.hindawi.com
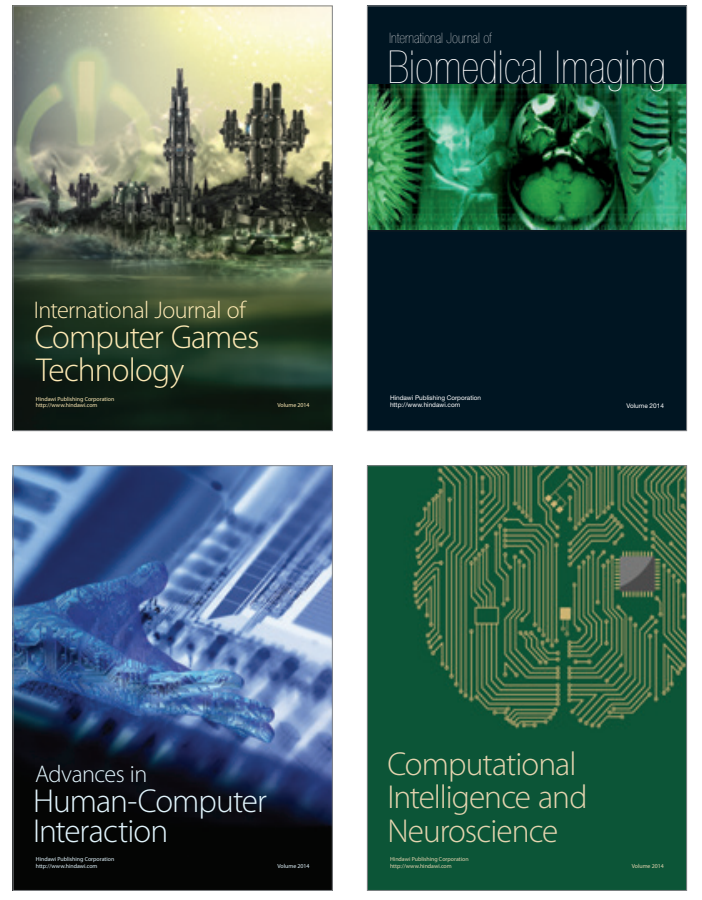
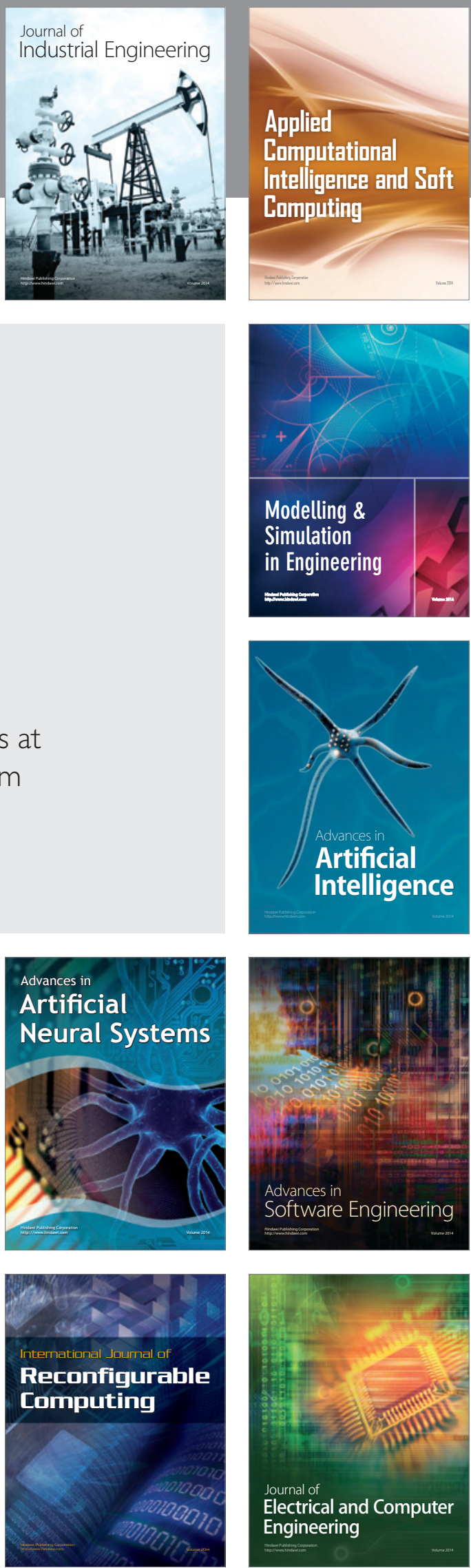